\title{
Microlensing of Quasars by Stars in Their Damped Lya Absorbers
}

\section{Citation}

Perna, Rosalba, and Abraham Loeb. 1997. "Microlensing of Quasars by Stars in Their Damped Lya Absorbers." The Astrophysical Journal 489 (2): 489-500. https://doi.org/10.1086/304806.

\section{Permanent link}

http://nrs.harvard.edu/urn-3:HUL.InstRepos:41393161

\section{Terms of Use}

This article was downloaded from Harvard University's DASH repository, and is made available under the terms and conditions applicable to Other Posted Material, as set forth at http:// nrs.harvard.edu/urn-3:HUL.InstRepos:dash.current.terms-of-use\#LAA

\section{Share Your Story}

The Harvard community has made this article openly available.

Please share how this access benefits you. Submit a story.

\section{Accessibility}


The AstrophysiCAL JouRnAL, 489:489-500, 1997 November 10

(C) 1997. The American Astronomical Society. All rights reserved. Printed in U.S.A

\title{
MICROLENSING OF QUASARS BY STARS IN THEIR DAMPED Ly $\alpha$ ABSORBERS
}

\author{
Rosalba Perna AND Abraham LOEB \\ Department of Astronomy, Harvard University, Mail Stop 46, 60 Garden Street, Cambridge, MA 02138 \\ Received 1997 January 29; accepted 1997 June 24
}

\begin{abstract}
The damped Ly $\alpha$ absorbers (DLAs) in quasar spectra are believed to be the progenitors of present-day disk galaxies. We examine the probability for microlensing of background quasars by stars in their DLAs. Microlensing by an individual star should magnify the continuum but not the broad emission lines of the quasars. Consequently, the equivalent width distribution of microlensed quasars would be distorted. We model a representative spiral galaxy as a closed system composed of a bulge, a disk, and a halo, and we evolve the mass fraction of stars in the disk based on the observed metallicity of DLAs at high redshifts. The microlensing signatures are stronger if the halo of the galaxy is made of massive compact halo objects (MACHOs). In this case, the distortion imprinted by microlensing on the equivalent width distribution of quasar emission lines can be detected with high significance in a sample of $\sim 10$ DLAs with $\mathrm{H}$ I column densities $N \gtrsim 10^{21} \mathrm{~cm}^{-2}$ and absorption redshifts $z_{\text {abs }} \lesssim 1$. About one-tenth of all quasars with DLAs $\left(N \gtrsim 10^{20} \mathrm{~cm}^{-2}\right)$ might show excess variability on timescales shorter than $5 \mathrm{yr}$. A search for these signals would complement microlensing searches in local galaxies and calibrate the MACHO mass fraction in galactic halos at high redshifts.
\end{abstract}

Subject headings: cosmology: theory — gravitational lensing — quasars: absorption lines

\section{INTRODUCTION}

Damped Ly $\alpha$ absorbers (DLAs) are thought to be the progenitors of present-day disk galaxies (Wolfe 1995). The abundance of heavy elements at low-ionization stages in the absorbers and the velocity field traced by them are consistent with values expected for disk galaxies (Turnshek et al. 1989; Wolfe et al. 1993; Lu et al. 1993; Pettini et al. 1994; Lu \& Wolfe 1994). Recent HIRES observations on the Keck telescope indicate that the weak, low-ionization, metal absorption lines in these systems often show the highest column density component at the edges of the velocity profile, as expected for absorption by a rotating gaseous disk (Wolfe 1995; Prochaska \& Wolfe 1997). Observations of redshifted $21 \mathrm{~cm}$ absorption and emission from DLAs indicate disklike structures of galactic dimensions (Briggs et al. 1989; Wolf, Lanzetta, \& Oren 1992a; Wolfe et al. 1992b; Briggs, Brinks, \& Wolfe 1997), and Faraday rotation observations are consistent with the existence of microgauss magnetic fields in these systems (Wolfe et al. 1992a; Welter, Perry, \& Kronberg 1984; Perry, Watson, \& Kronberg 1993).

Since the total comoving density of $\mathrm{H} \mathrm{I}$ in DLAs is comparable to that of stars in the local universe, it is only natural to postulate that the cold gas out of which most of the present population of stars had formed was already assembled in galaxies at $z \approx 3$ (Lanzetta, Wolfe, \& Turnshek 1995; Wolfe et al. 1995; Storrie-Lombardi, Irwin, \& McMahon 1996a; Storrie-Lombardi et al. 1996b). However, the typical metallicity of $Z \approx 0.1 Z_{\odot}$ (Smith et al. 1996 and Lu et al. 1996, and references therein) and the dust-to-gas ratio (Fall \& Pei 1996; Pei, Fall, \& Bechtold 1991) in these systems imply that star formation was only at its infancy at these early times. This assertion is consistent with recent determinations of the star formation rate at high redshifts (Madau 1996 and Lowenthal et al. 1997, and references therein). Since star formation requires cold gas, and since most of the $\mathrm{H}$ I detected through Ly $\alpha$ absorption lines are in damped systems, DLAs are the natural sites for star formation at high redshifts (Fall \& Pei 1996). In order to unravel the star formation history of the universe, it is of fundamental importance to probe not only the gas in DLAs but the stars as well.

Direct images of distant DLAs typically reveal a luminous galactic core that is separated by $10-20 \mathrm{kpc}$ from the line of sight to the quasar (Steidel et al. 1994, 1995, 1996). However, any inference about the fraction of gas converted into stars in these systems requires a prior knowledge of the initial mass function of these stars and their formation history. In addition, a major fraction of the baryonic mass might reside in the outer low surface brightness halos of these systems. This possibility is raised by recent microlensing searches (Alcock et al. 1997), which indicate that a nonnegligible fraction of the massive halo of the Milky Way might exist in the form of massive compact halo objects (MACHOs). As a supplement to local microlensing searches, it would be particularly interesting to examine whether MACHOs populate the halos of galaxies at high redshifts. While the microlensing probability is only $\sim 10^{-6}$ in the Milky Way halo ( $\sim 10 \mathrm{kpc})$, its value increases up to unity in the cores of halos at cosmological distances $(\sim 5 \mathrm{Gpc})$. This follows from the linear dependence of the lensing cross section on the observer lens distance for a source at infinity. Near the center of a DLA, macrolensing by the entire galactic potential might take place and yield widely separated quasar images; the likelihood of macrolensing and its generic signatures were discussed recently by Bartelmann \& Loeb (1996) and by Perna, Loeb, \& Bartelmann (1997).

In this paper, we quantify the expected microlensing probability in distant spiral galaxies that show up as DLAs in quasar spectra. The obvious advantage of these galactic systems is that they are selected based on their proximity on the sky to a compact source of light in the form of a quasar. Detection of microlensing of the quasar can be used to probe the stellar mass fraction of DLAs and to test whether the halos of galaxies at high redshifts are made of MACHOs. The characteristic Einstein radius of a solar mass lens at a cosmological distance is $\sim 5 \times 10^{16} \mathrm{~cm}$, comfortably in between the scales of the continuum-emitting 
accretion disk $\left(\lesssim 10^{15} \mathrm{~cm}\right)$ and the broad-line region $\left(\sim 3 \times 10^{17} \mathrm{~cm}\right)$ of a bright quasar. This implies that a single microlensing event would magnify the continuum but not the broad lines emitted by the quasar. The lines would only be affected by the macrolensing effect of the galaxy as a whole. As a result of this differential amplification, the equivalent width distribution of the broad emission lines (Francis 1993) will be significantly distorted in a sample of microlensed quasars. Microlensing would also result in excess variability of such quasars. In the following, we will quantify the above signatures for a model spiral galaxy that acts as a high-redshift DLA.

The existence of a population of normal disk galaxies at high redshifts is supported by recent Keck HIRES spectra of the damped Ly $\alpha$ absorber toward the quasar Q2233+1310 (Lu, Sargent, \& Barlow 1997). The metal absorption lines of the absorbing galaxy are shifted relative to its Ly $\alpha$ emission redshift (Djorgovski et al. 1996), indicating a rotation velocity $\geq 200 \mathrm{~km} \mathrm{~s}^{-1}$ at a separation $\sim 20$ kpc. Here we limit our attention to galaxies of this population. For concreteness, we focus our discussion on a single spiral galaxy and extrapolate its present-day properties back in time, using the simplest closed box model for its baryonic content. Based on the observed metallicity of DLAs, we assume that the stars constitute only $10 \%$ of the total disk mass at $z \approx 3$, and that $\mathrm{H} \mathrm{I}$ is gradually converted into stars until the present epoch. The galaxy is modeled as having three components: an old stellar bulge, a disk made of gas and stars, and a halo. We compute the significance of microlensing for each of these components. Our simplified model ignores the diversity among different disk galaxies or DLAs and focuses on a typical massive spiral galaxy. Its only purpose is to examine whether microlensing could be detected in this idealized case. Recent imaging of DLA galaxies (Le Brun, Bergeron, \& Boissé 1996) indicates that while some systems are clearly spirals, others have irregular morphologies. A more diverse treatment of this problem would involve many more free parameters and is beyond the scope of this paper. Our primary objective here is to motivate an observational search for these microlensing signatures, rather than to explore the entire possible range of parameter space.

The outline of the paper is as follows. In $\S 2$, we describe the distributions of $\mathrm{H}$ I and stars in our model for a galactic disk. These distributions are then used in $\S 3$ to compute the microlensing probability as a function of the intercepted $\mathrm{H}$ I column density of the disk. In $\S 4$, we repeat the calculation for a halo made of MACHOs. In $\S 5$, we relate these results to observable signatures. Finally, in $\S 6$, we summarize our main conclusions.

\section{DISTRIBUTIONS OF H I AND STARS IN MODEL DISK GALAXY}

The cross section for gravitational lensing of a highredshift source $\left(z_{s} \geq 2\right)$ peaks at intermediate redshifts, $z \sim 0.2-0.8$ (see, e.g., Fig. 1 in Refregier \& Loeb 1997). In this redshift range, there is compelling observational evidence indicating that disk galaxies have not accreted large quantities of matter. The evidence includes the low level of lopsidedness in spiral galaxies (Zaritzky \& Rix 1997), the thinness and coldness of the stellar distribution in galactic disks (Tóth \& Ostriker 1992), the small scatter in the TullyFisher relation (Eisenstein \& Loeb 1996), the similarity between the kinematics at a given luminosity of galaxies out to $z \sim 1$ and local galaxies (Vogt et al. 1996), and the statistics of multiply imaged quasars (Mao \& Kochanek 1994; Rix et al. 1994). We therefore anchor our modeling to the current properties of a typical galactic disk and evolve the gaseous mass fraction of its disk based on a simple closed box model.

Observations of local spiral galaxies show that their $\mathrm{H} \mathrm{I}$ gas is generically distributed in an exponential disk with a radial face-on column density profile (Broeils \& van Woerden 1994),

$$
N^{\text {face-on }}(R, \text { now })=N_{0} \exp \left(-R / R_{g}\right),
$$

where $N_{0}$ is the central column density and $R_{g}$ is the scale radius of the $\mathrm{H}$ I disk. For our model galaxy, we adopt $N_{0} \approx 10^{21} \mathrm{~cm}^{-2}$, which is the typical value observed in nearby galaxies.

The surface mass density of stars in a nearby spiral galaxy can be modeled as the sum of an exponential disk and a bulge, described by a de Vaucouleur law,

$$
\begin{aligned}
\Sigma_{*}^{\text {face-on }}(R, \text { now })= & \Sigma_{0} \exp \left(-R / R_{s}\right) \\
& +\Sigma_{e} \exp \left\{-7.67\left[\left(R / R_{e}\right)^{1 / 4}-1\right]\right\},
\end{aligned}
$$

where $R$ is the radial coordinate on the face of the disk. The central mass density of stars in the disk, $\Sigma_{0}$, can be calibrated from the characteristic surface brightness observed in galactic disks $I_{0} \approx 21.65 \mu_{B}$, where $\mu_{B}$ is $B$ magnitude per $\operatorname{arcsec}^{2}$ (Freeman 1970). We convert the surface brightness to mass density by assuming a mass-to-light ratio of 5 in solar units (Broeils \& Courteau 1997). The bulge surface density at $R=R_{e}, \Sigma_{e}$, can be related to $\Sigma_{0}$ and the total disk and bulge luminosities, $L_{d}$ and $L_{b}$, through the relation $L_{d} / L_{b}=0.28\left(R_{s} / R_{e}\right)^{2}\left(\Sigma_{0} / \Sigma_{e}\right)$. This relation results from an integral of the corresponding surface brightnesses over the area of the disk. For typical values of $L_{d} / L_{b} \approx 1.3$ and $R_{e} / R_{s} \approx 0.4$ (Freeman 1970; Burstein 1979), we get $\Sigma_{e} / \Sigma_{0} \approx$ 1.5. The scale radius of the stars $R_{s}$ is strongly correlated with the scale radius of the gas $R_{g}$. Figure $8 b$ in Broeils \& van Woerden (1994) illustrates that the H I surface densities of different galaxies fall off with very similar profiles when the radius is expressed in units of the optical radius $R_{25}$ of the $25 \mathrm{mag} \operatorname{arcsec}^{-2}$ isophote in the $B$ band. With $I_{0} \approx$ $21.65 \mu_{B}$, we derive $R_{25} \approx 3.1 R_{s}$, and from the slopes of the profiles in Broeils \& van Woerden (1994), we estimate $R_{g} \approx$ $0.8 R_{25}$ so that $R_{s} \approx 0.4 R_{g}$. For our model galaxy, we adopt the typical value of $R_{g}=10 \mathrm{kpc}$. Our results for microlensing by stars in the disk are not very sensitive to the particular choice of $R_{g}$ and depend mostly on the adopted ratio between $R_{g}$ and $R_{s}$, for which the observed scatter is small.

We evolve the model galaxy back in time as a closed system. We assume that the model disk was already assembled at $z \approx 3$ and that its $\mathrm{H}$ i content evolved only as a result of star formation; this assumption is consistent with the fact that the comoving $\mathrm{H}$ I density of DLAs at $z \approx 3$ is comparable to the mass density of stars in the local universe. Since nearby galaxies exhibit a much older stellar population in their bulges than in their disks, we keep the bulge properties fixed in time and evolve only the stellar mass fraction of the disk with redshift for $z \lesssim 3$. Based on the observed metallicity of DLAs (Pettini et al. 1994, 1997), we assume that at a redshift $z \approx 3$, the mass fraction of stars in the disk was about one-tenth of its present value. Under 
these assumptions, equation (1) is generalized to

$$
N^{\text {face-on }}(R, z)=N_{0} \exp \left(-R / R_{g}\right)+\Delta N_{0}(z) \exp \left(-R / R_{s}\right),
$$

where, in the closed box model, the $\mathrm{H}$ I and stellar surface densities obey

$$
\Sigma_{\mathrm{HI}}^{\mathrm{face-on}}(R, z)+\Sigma_{*}^{\mathrm{face-on}}(R, z)=\mathrm{const},
$$

with $\Sigma_{\mathrm{HI}}=m_{\mathrm{H}} N$, and therefore

$$
\Delta \Sigma_{\mathrm{HI}}(R, z)=\Sigma_{*, \mathrm{disk}}(R, 0)-\Sigma_{*, \mathrm{disk}}(R, z) .
$$

Here $m_{\mathrm{H}}$ is the mass of a hydrogen atom, and the subscript "*, disk" refers to the disk stars only. In the context of the closed box model, the gas out of which the disk stars formed must have had the same radial distribution as those stars have today, and so equation (5) can be regarded as a condition on the corresponding central densities. We constrain the redshift evolution of $\Sigma_{\mathrm{H} \text { I }}$ by the condition $\Sigma_{* \text {, disk }}(z=$ $3) \approx 10 \% \Sigma_{* \text {,disk }}(z=0)$, and by the observed redshift evolution of the H I content of DLAs (Wolfe et al. 1995; StorrieLombardi et al. 1996a). Both of these constraints are reasonably satisfied in the redshift interval $0 \lesssim z \lesssim 3$, if one uses a power-law redshift evolution of the form $\Delta \Sigma_{\mathrm{HI}}(R, z)=m_{\mathrm{H}} \tilde{N} z^{\delta} \exp \left(-R / R_{s}\right)$, with $\delta \approx 6$ and $\tilde{N}=$ $10^{20} \mathrm{~cm}^{-2}$. Based on equation (5), we then derive

$$
\begin{aligned}
\Sigma_{*, \text { disk }}(R, z) & =\left[\Sigma_{0}-m_{\mathrm{H}} \Delta N_{0}(z)\right] \exp \left(-R / R_{s}\right) \\
& \equiv \Sigma_{*}^{\prime}(z) \exp \left(-R / R_{s}\right),
\end{aligned}
$$

and thus the stellar surface density profile is given by

$$
\begin{aligned}
\Sigma_{*}^{\mathrm{face}-\mathrm{on}}(R, z)= & \Sigma_{*}^{\prime}(z) \exp \left(-R / R_{s}\right) \\
& +\Sigma_{e} \exp \left\{-7.67\left[\left(R / R_{e}\right)^{1 / 4}-1\right]\right\} .
\end{aligned}
$$

Equations (3) and (7) describe the radial profiles for a face-on disk. If the normal to the disk is inclined by an angle $\theta$ with respect to the line of sight, then the observed distributions are changed to

$N(R, \theta, z)$

$$
=\frac{\left[N_{0} \exp \left(-R / R_{g}\right)+\Delta N_{0}(z) \exp \left(-R / R_{s}\right)\right]}{\cos \theta},
$$

and

$$
\begin{array}{r}
\Sigma_{*}(R, \theta, \phi, z)=\Sigma_{*, \text { disk }}+\Sigma_{*, \text { bulge }}=\frac{\Sigma_{*}^{\prime}(z) \exp \left(-R / R_{s}\right)}{\cos \theta} \\
+\Sigma_{e} \exp \left\{-7.67\left[\left(b / R_{e}\right)^{1 / 4}-1\right]\right\}
\end{array}
$$

where $b=R\left(1-\sin ^{2} \theta \cos ^{2} \phi\right)^{1 / 2}$ is the impact parameter and $\phi$ is the azimuthal coordinate on the sky of the line of sight relative to the center of the galaxy. In this derivation, we have assumed, for simplicity, a razor thin disk and a spherical bulge.

\section{MICROLENSING BY STARS IN THE DISK AND THE BULGE}

For a single galaxy, the optical depth to microlensing is given by the dimensionless surface mass density of its stars,

$$
\kappa_{*} \equiv \frac{\Sigma_{*}}{\Sigma_{\mathrm{crit}}}
$$

as normalized by the critical surface density,

$$
\Sigma_{\mathrm{crit}}=\frac{c^{2} D_{s}}{4 \pi G D_{d} D_{\mathrm{ds}}} .
$$

Here $G$ is the gravitational constant, $c$ is the speed of light, and $D_{d}, D_{s}$, and $D_{\mathrm{ds}}$ are the angular diameter distances between the observer and the lens, the observer and the source, and lens and the source, respectively (Schneider, Ehlers, \& Falco 1992). Throughout the paper, we assume $H_{0}=50 \mathrm{~km} \mathrm{~s}^{-1} \mathrm{Mpc}^{-1}, \Omega_{0}=1$, and a quasar redshift of $z_{s}=4$.

We would like to find the probability distribution for the microlensing optical depth, $P\left(\kappa_{*}\right)$, given a particular observed value for the $\mathrm{H}$ I column density in our model galaxy. We assume random inclinations of the galactic disk relative to the line of sight. Given the $\mathrm{H}$ I column density in equation (8), the inclination angle is restricted by the condition $\theta \geq \theta_{0}$, where

$$
\cos \theta_{0} \equiv \gamma_{0}=\min \left[1, \frac{N_{0}+\Delta N_{0}(z)}{N}\right] .
$$

The uniform probability distribution for the cosine of the inclination angle is then

$$
P(\cos \theta) d \cos \theta=\frac{1}{\gamma_{0}} d \cos \theta \text { for } 0 \leq \cos \theta \leq \gamma_{0} .
$$

Given an inclination angle, $\theta$, the microlensing optical depth $\kappa_{*}$ can be obtained from equations (9) and (10). The value of $\kappa_{*}$ depends on the observed column density $N$ through the radius $R$ intercepted by the line of sight in the plane of the disk; this radius in turn is obtained by inverting equation (8). Given an observed $\mathrm{H}$ I column density $N$, the probability distribution for the optical depth $\kappa_{*}$ is

$$
\begin{aligned}
P\left(\kappa_{*} \mid N\right)= & \frac{1}{2 \pi \gamma_{0}} \int_{0}^{2 \pi} d \phi\left|\frac{d \cos \theta}{d \kappa_{*}}\right| \\
= & \frac{1}{2 \pi \gamma_{0}} \int_{0}^{2 \pi} d \phi \mid-1.975 \kappa_{b} b^{-3 / 4} R_{e}^{-1 / 4} \frac{d b}{d \cos \theta} \\
& \quad-\left.\frac{\kappa_{d}}{\cos ^{2} \theta}\left(1+\frac{\cos \theta}{R_{s}} \frac{d R}{d \cos \theta}\right)\right|^{-1}
\end{aligned}
$$

with

$$
\begin{aligned}
& \frac{d R}{d \cos \theta} \\
& \quad=-\frac{N}{R_{g}^{-1} N_{0} \exp \left(-R / R_{g}\right)+R_{s}^{-1} \Delta N_{0}(z) \exp \left(-R / R_{s}\right)},
\end{aligned}
$$

where $\kappa_{b}=\Sigma_{\text {bulge }} / \Sigma_{\text {crit }}$ and $\kappa_{d}=\Sigma_{\text {disk }} / \Sigma_{\text {crit }}$. Note that $P\left(\kappa_{*} \mid N\right)$ depends on the absorber redshift through equations (10), (11), (12), and (15).

If, in total, $\kappa \gtrsim 1$, then the galaxy as a whole produces multiple images. Under these circumstances, there could still be microlensing, as observed in the lens Q2237+0305 (Irwin et al. 1989; Wambsganss, Paczyński, \& Schneider 1990; Rauch \& Blandford 1991; Racine 1991). However, in this case, the observed spectrum will not show a single 
column density since different quasar images intercept the disk at different impact parameters and therefore probe different $\mathrm{H}$ I column densities. The Ly $\alpha$ absorption feature will then be composed of multiple troughs, each having a depth proportional to the magnification of its corresponding image and a width scaling as the square root of the $\mathrm{H} \mathrm{I}$ column density probed by this image (Bartelmann \& Loeb 1996; Loeb 1997). Since the interpretation of $N$ is not unique in this case, and since most DLAs with $N \gtrsim 10^{20}$ $\mathrm{cm}^{-2}$ are not macrolensed (Bartelmann \& Loeb 1996), we restrict our attention to values of $\kappa_{*} \lesssim 1$. Figure 1 shows the probability at a given value of $N$ for obtaining a value of $\kappa_{*}$ smaller than unity but higher than some threshold $\kappa_{\min }$, $P\left(\kappa_{\min }<\kappa_{*}<1 \mid N\right)=\int_{\kappa_{\min }}^{1} d \kappa_{*} P\left(\kappa_{*} \mid N\right)$. The different panels correspond to different redshifts of our model galaxy; Fig. $1 a$ refers to $z_{\text {abs }}=0.5$, and Fig. $1 b$ corresponds to $z_{\text {abs }}=2.5$. For a low absorber redshift, the probability of having a microlensing optical depth $\kappa_{*}>\kappa_{\min }=0.05$ is negligible at low column densities $\left(N \lesssim 3 \times 10^{20} \mathrm{~cm}^{-2}\right)$ but becomes significant above $10^{21} \mathrm{~cm}^{-2}$. For a high absorber redshift, the probability of having $\kappa_{*}>0.05$ becomes significant at yet higher column densities, $N \gtrsim(4-9) \times 10^{21}$ $\mathrm{cm}^{-2}$. This is due to the increase in $\Sigma_{\text {crit }}$ as the lens gets closer to the source. In order to reach the same values of $\kappa_{*}$ at high redshifts, it is necessary that the line of sight to the quasar pass closer to the center of the absorber, where the $\mathrm{H}$ I density is higher. The drop in the probabilities at yet higher $\mathrm{H} \mathrm{I}$ densities is due to the increase in the probability of finding systems with $\kappa>1$ (which we exclude from our analysis).

\section{MICROLENSING BY MACHOS}

We now consider the possibility that in addition to the stars in the disk and the bulge, there is a population of compact objects that dominate the mass of the halo in our model galaxy.

We model the spherically averaged mass distribution of the entire galaxy as a singular isothermal sphere (SIS) with a one-dimensional velocity dispersion $\sigma$,

$$
M_{\mathrm{SIS}}(r)=\frac{2 \sigma^{2}}{G} r
$$

where $r$ is the radial spherical coordinate and $\sigma$ is the velocity dispersion for which we adopt a typical value of $170 \mathrm{~km}$ $\mathrm{s}^{-1}$. The SIS model yields a flat rotation curve, as observed in nearby spirals. We assume that the total mass of the galaxy is composed of the disk of gas and stars (cf. eqs. [3] and [7]) and a spherical component (denoted by SP) that includes both the bulge and the halo,

$$
M_{\mathrm{SIS}}(r)=M_{\mathrm{SP}}(r)+M_{\mathrm{H} \mathrm{I} \text {, disk }}(r)+M_{*, \text { disk }}(r) .
$$

The $\mathrm{H}$ I and stellar masses out to a radius $R=r$ on the face of the disk are found by integrating their respective surface

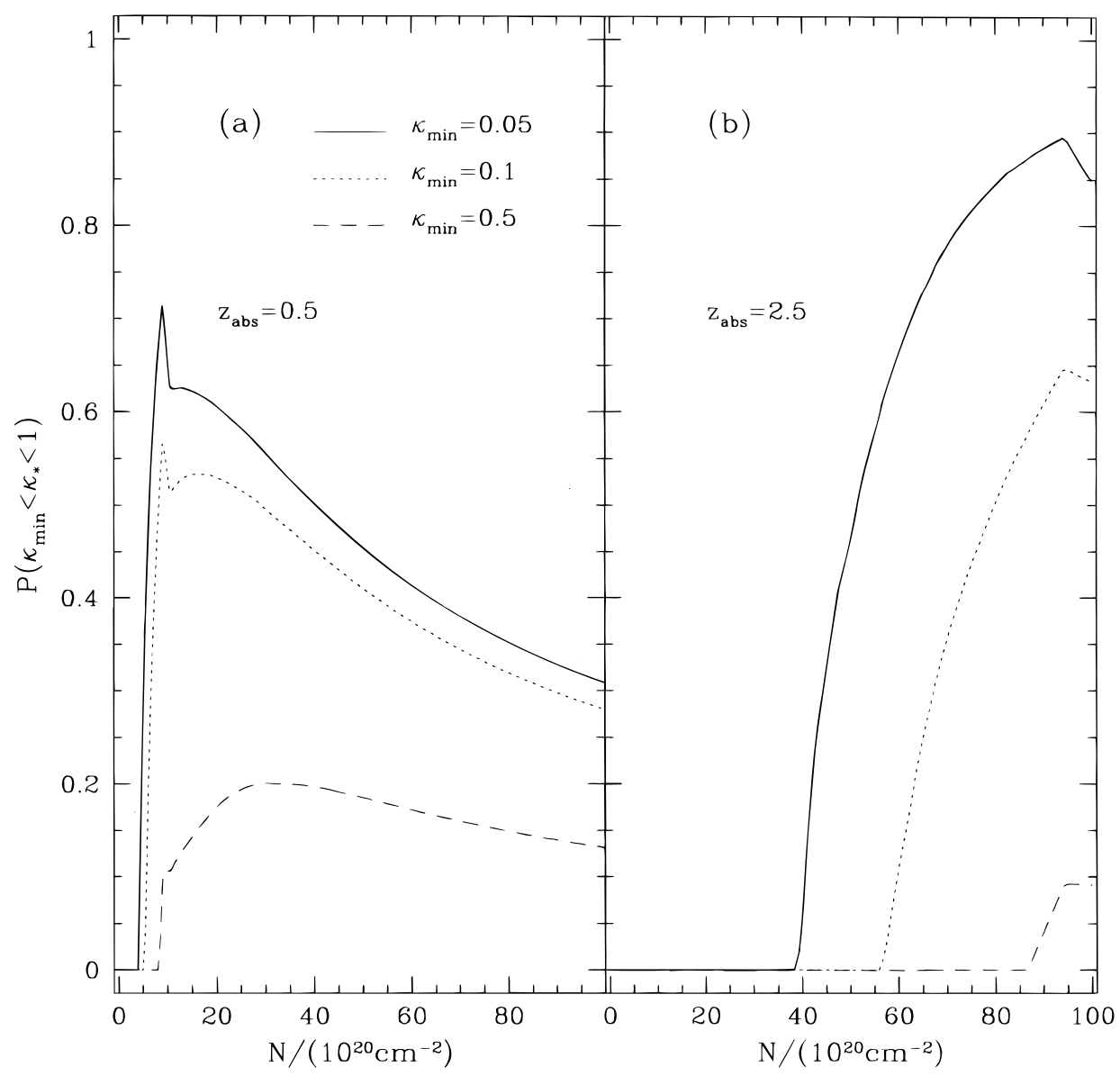

FIG. 1.-Probability of finding the microlensing optical depth $\kappa_{*}$ in the range between $\kappa_{\min }$ and 1 as a function of the observed $\mathrm{H}$ I column density in our model galaxy. Results are shown for an absorber (lens) redshift $(a) z_{\text {abs }}=0.5$ and $(b) z_{\text {abs }}=2.5$. In both panels, we show three curves for $\kappa_{\min }=0.05$ (solid line), $\kappa_{\min }=0.1$ (dotted line), and $\kappa_{\min }=0.5$ (dashed line). The source redshift is fixed at $z_{s}=4$. 


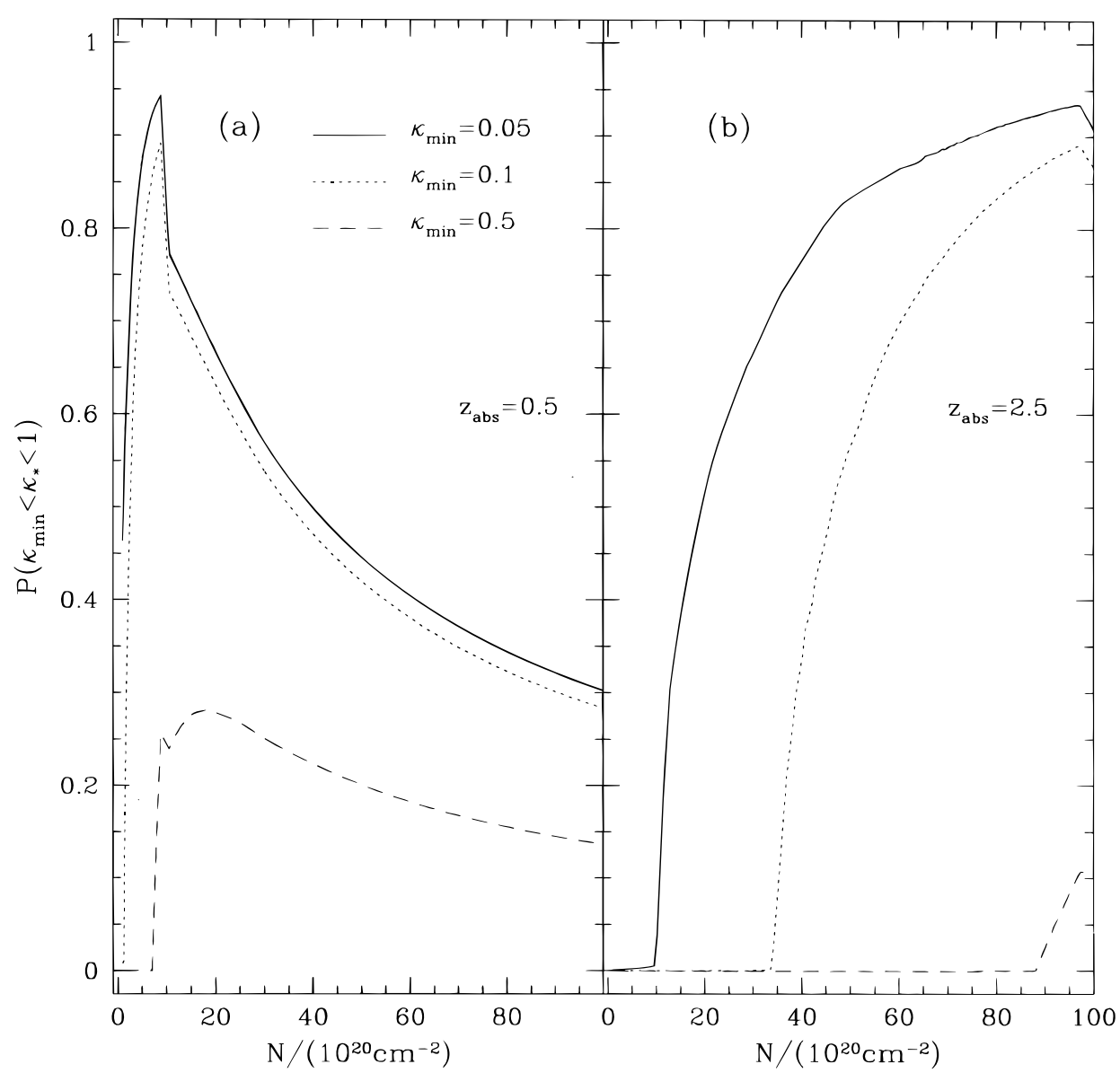

FIG. 2.- Same as Fig. 1, but including a halo of MACHOs in addition to the bulge and the disk stars. The total microlensing optical depth is $\kappa_{*}=\kappa_{* \text {,disk }}+\kappa_{\mathrm{SP}}$ (cf. eqs. [22] and [23]).

densities,

$$
\begin{aligned}
M_{\mathrm{H} \mathrm{I}, \text { disk }}(r) & =2 \pi \int_{0}^{r} \Sigma_{\mathrm{HI}}^{0} \exp \left(-\frac{R}{R_{g}}\right) R d R \\
& =2 \pi \Sigma_{\mathrm{HI}}^{0} R_{g}^{2}\left[1-\exp \left(-\frac{r}{R_{g}}\right)\left(1+\frac{r}{R_{g}}\right)\right],
\end{aligned}
$$

with $\Sigma_{\mathrm{HI}}^{0}=m_{\mathrm{H}} N_{0}$ and

$$
M_{*, \text { disk }}(r)=2 \pi \Sigma_{0} R_{s}^{2}\left[1-\exp \left(-\frac{r}{R_{s}}\right)\left(1+\frac{r}{R_{s}}\right)\right] .
$$

The mass density of the spherical component (bulge plus halo) can be derived from equation (17) through the relation

$$
\begin{aligned}
\rho_{\mathrm{SP}}(r) & =\frac{1}{4 \pi r^{2}} \frac{d M_{\mathrm{SP}}}{d r} \\
& =\frac{\sigma^{2}}{2 \pi G r^{2}}-\frac{1}{2 r}\left[\Sigma_{\mathrm{HI}}^{0} \exp \left(-\frac{r}{R_{g}}\right)+\Sigma_{0} \exp \left(-\frac{r}{R_{\mathrm{s}}}\right)\right] .
\end{aligned}
$$

The surface density as a function of the impact parameter on the sky $b$ is then found by integrating this mass density along the line of sight $\Sigma(b)=\int_{-\infty}^{\infty} d \zeta \rho(b, \zeta)$ with $r=\left(b^{2}+\zeta^{2}\right)^{1 / 2}$. This yields

$$
\Sigma_{\mathrm{SP}}(b)=\frac{\sigma^{2}}{2 G b}-\frac{\Gamma(1 / 2)}{\sqrt{\pi}}\left[\Sigma_{\mathrm{HI}}^{0} K_{0}\left(\frac{b}{R_{g}}\right)+\Sigma_{0} K_{0}\left(\frac{b}{R_{s}}\right)\right],
$$

where $K_{0}$ is the modified Bessel function of the zeroth order. The optical depth to microlensing is then given by

$$
\begin{aligned}
\kappa(R, \theta, \phi) & =\kappa_{\mathrm{SP}}+\kappa_{* \text {, disk }} \\
& \equiv \frac{1}{\Sigma_{\text {crit }}}\left[\Sigma_{\mathrm{SP}}(b)+\frac{\Sigma_{0} \exp \left(-R / R_{s}\right)}{\cos \theta}\right],
\end{aligned}
$$

where, as before, $\theta$ is the inclination of the normal to the disk relative to the line of sight, $\phi$ is the azimuthal coordinate on the sky, $R$ is the radial coordinate in the plane of the disk, and $b=R\left(1-\sin ^{2} \theta \cos ^{2} \phi\right)^{1 / 2}$. The probability distribution of $\kappa_{*}$ for an observed $\mathrm{H}$ I column density $N$ can be calculated again as in equation (14),

$$
\begin{aligned}
P\left(\kappa_{*} \mid N\right)= & \frac{1}{2 \pi \gamma_{0}} \int_{0}^{2 \pi} d \phi \mid \frac{d b}{d \cos \theta}\left[-\frac{\kappa_{\mathrm{SIS}}^{0}}{b^{2}}\right. \\
& \left.+\frac{\kappa_{* \text {, disk }}^{0}}{R_{s}} K_{1}\left(\frac{b}{R_{s}}\right)+\frac{\kappa_{\mathrm{HI}}^{0}}{R_{g}} K_{1}\left(\frac{b}{R_{g}}\right)\right] \\
& -\left.\frac{\kappa_{* \text {, disk }}}{\cos ^{2} \theta}\left(1+\frac{\cos \theta}{R_{s}} \frac{d R}{d \cos \theta}\right)\right|^{-1},
\end{aligned}
$$

where $K_{1}$ is the modified Bessel function of the first order and $d R / d \cos \theta$ is given in equation (15).

Figure 2 shows the probability for obtaining a value of $\kappa_{*}$ smaller than unity but higher than some threshold $\kappa_{\min }$ at either a low absorber redshift $\left(z_{\text {abs }}=0.5\right.$ in Fig. $\left.2 a\right)$ or a high absorber redshift $\left(z_{\text {abs }}=2.5\right.$ in Fig. $\left.2 b\right)$. As evident from 
comparing Figures 1 and 2, the existence of MACHOs enhances considerably the microlensing probability in DLAs that are not macrolensed. This is especially true for absorbers with lower column densities.

\section{OBSERVATIONAL SIGNATURES OF MICROLENSING}

The microlensing probability derived in the previous sections can be related to two observable signatures: (i) distortion in the equivalent width distribution of the broad emission lines of microlensed quasars (Canizares 1982) and (ii) excess variability of microlensed quasars (see, e.g., Wambsganss \& Kundić 1995). In the following, we quantify both of these effects as a function of the observed $\mathrm{H} \mathrm{I}$ column density for our model galaxy.

\subsection{Equivalent Width Distribution of Microlensed Quasars}

Quasars can be affected significantly by microlensing only if the size of their emission region is smaller than the projected size $r_{\mathrm{E}}$ of the lensing zone, i.e., the "Einstein radius" of the lensing star. The maximum magnification of a circular source of radius $r_{s}$ and uniform brightness is given by (Schneider et al. 1992, p. 38)

$$
\mu_{\max }=\sqrt{1+4\left(r_{\mathrm{E}} / r_{s}\right)^{2}} .
$$

At cosmological distances, the lensing zone of a star of mass $M_{\text {star }}$ has a characteristic scale of $\sim 5 \times 10^{16}\left(M_{\text {star }} / M_{\odot}\right)^{1 / 2}$ $\mathrm{cm}$. In comparison, the optical continuum emission in quasars is believed to originate from a compact accretion disk. The UV bump observed in quasar spectra is often associated with thermal emission from an accretion disk with a surface temperature $T_{\text {disk }} \equiv 10^{5} T_{5} \mathrm{~K}$, where $T_{5} \sim 1$ (e.g., Laor 1990), and so the scale of the disk emission region must be $\sim 10^{15} \mathrm{~cm} T_{5}^{-2} L_{46}^{1 / 2}$, where $L_{46}$ is the corresponding luminosity of the quasar in units of $10^{46} \mathrm{ergs}^{-1}$. Thus, for lens masses $M_{\text {star }} \gg 10^{-3} M_{\odot}$, the continuum source is much smaller than the lensing zone and therefore could be magnified considerably. This expectation is indeed confirmed in the nearby lens of Q2237+0305, where variability due to microlensing has been observed (Wambsganss et al. 1990; Rauch \& Blandford 1991; Racine 1991; see also Gould \& Miralda-Escudé 1996). However, reverberation studies of the time lag between variations in the continuum and the line emission in active galactic nuclei indicate that the broad emission lines of quasars originate at a distance of $\sim 3 \times 10^{17} \mathrm{~cm} \mathrm{~L}_{46}^{1 / 2}$ (Peterson 1993; Maoz 1997). For a solar mass lens with luminosity $10^{46} \mathrm{ergs}^{-2}$, equation (24) implies that the maximum magnification differs from unity by only a few percent. This argues that microlensing of the broad-line region by a single star can be safely ignored. The broad-line region would only be macrolensed by a factor $\langle\mu\rangle$ because of the average effect of all the stars. As a result of the difference in amplification between the lines and the continuum, the equivalent width of the lines will change during a microlensing event. For compact objects that are uniformly distributed throughout the universe, the microlensing optical depth is a function of redshift and therefore should result in an apparent evolution of the equivalent width distribution of quasars. Therefore, the lack of such evolution in existing quasar samples was used to set an upper limit on the mean cosmological density of stars and MACHOs in the universe (Dalcanton et al. 1994). Nemiroff (1988) examined the effect of microlensing on the shape of the broad emission lines, and Hawkins (1996) addressed the potential to detect cosmologically distributed microlenses based on quasar variability data. Here we propose to look for microlensing signatures through a comparison between the equivalent width distribution of quasars with DLAs and those without. Since quasars with DLAs are selected based on their small projected separation from foreground galaxies, they are more likely to be lensed by stars or MACHOs than the rest of the quasar population. The latter approach is likely to have a larger signalto-noise ratio if most of the stellar objects in the universe are grouped into galaxies. Since DLAs appear only for a small fraction of all quasars, $\sim 10 \%\left(N / 10^{20} \mathrm{~cm}^{-2}\right)^{-1 / 2}$, it should be straightforward to obtain an accurate equivalent width (EW) distribution for a control sample of quasars that are not microlensed.

In order to find the effect of microlensing on the EW distribution of quasar emission lines, it is necessary to calculate the magnification probability as a function of $\kappa_{*}$. This probability function was derived in the literature through extensive numerical simulations (Witt 1993; Wambsganss 1990; Lewis et al. 1993; Kundić \& Wambsganss 1993; Wambsganss \& Kundić 1995). In particular, the simulations of Rauch et al. (1992) demonstrated that the probability distribution of magnifications for a point source seen through a field of stars can be reasonably well approximated by the analytic expression proposed by Peacock (1986):

$$
P(\mu, \chi) d \mu=\exp [f(\mu, \chi)] \frac{d f}{d \mu}
$$

where

$$
f(\mu)=2 \chi\left[1-\mu\left(\mu^{2}-1\right)^{-1 / 2}\right]
$$

and

$$
d f / d \mu=2 \chi\left(\mu^{2}-1\right)^{-3 / 2},
$$

and the parameter $\chi$ is related to the dimensionless surface mass density $\kappa_{*}$ through the equation

$$
\langle\mu\rangle \equiv \int_{1}^{\infty} \mu P(\mu, \chi) d \mu=\frac{1}{\left(1-\kappa_{*}\right)^{2}} .
$$

The probability distribution of magnifications for a given value of the observed $\mathrm{H}$ I column density $N$ can be obtained by combining the probability distribution for $\kappa_{*}$ at a given $N$ (cf. eq. [14] or eq. [23]) with the probability distribution of magnifications at a given $\kappa_{*}$ (cf. eq. [25]),

$$
P(\mu \mid N)=\int_{0}^{1} d \kappa_{*} P\left(\mu \mid \kappa_{*}\right) P\left(\kappa_{*} \mid N\right) .
$$

We define $I_{v}\left(\lambda_{0}\right)$ as the intensity of the quasar continuum in the neighborhood of the wavelength $\lambda_{0}$ of a particular emission line, and we define $\Delta I_{v}(\lambda)$ as the difference between the total measured intensity and the continuum. If the continuum is magnified by a factor $\mu$, then its observed intensity changes to $\mu I_{v}\left(\lambda_{0}\right)$. The extended broad-line region is affected by the combined effect of many stars, and so the intensity of the lines is enhanced by the average magnification factor $\langle\mu\rangle$ in equation (26). Consequently, the EW of the emission line, defined as

$$
W_{\lambda} \equiv \int \frac{\Delta I_{v}}{I_{v}\left(\lambda_{0}\right)} d \lambda
$$


is changed by a factor $\langle\mu\rangle / \mu$, namely,

$$
W_{\lambda}=W_{0} \frac{\langle\mu\rangle}{\mu},
$$

where $W_{0}$ is the intrinsic $\mathrm{EW}$ of the unlensed quasar.

Even in the absence of lensing, quasars do not possess a unique EW value in their emission lines, but rather they show a wide probability distribution of EW values (Francis 1993), which we define as $P\left(W_{0}\right)$. The magnification due to lensing distorts this probability distribution. The modified distribution of EW values for quasars that show DLA absorption with a column density $N$ would be

$$
P\left(W_{\lambda} \mid N\right)=\int_{1}^{\infty} d \mu P(\mu \mid N) P\left(\frac{\mu}{\langle\mu\rangle} W_{\lambda}\right) \frac{\mu}{\langle\mu\rangle} .
$$

The observed probability distribution of EW can be well approximated as a log Gaussian function (see also Dalcanton et al. 1994),

$$
P\left(W_{0}\right)=\frac{1}{\sqrt{2 \pi \sigma_{W}^{2}} W_{0}} \exp \left\{-\frac{\left[\ln \left(W_{0}\right)-\omega\right]^{2}}{2 \sigma_{W}^{2}}\right\},
$$

where the parameters $\omega$ and $\sigma_{W}$ obtain different values for different emission lines. We optimize these values so as to fit the data from the Large Bright Quasar Survey (LBQS) reported by Francis (1993).

Figures 3 and 4 illustrate the unlensed distributions (solid lines) chosen to best represent the observational data from the LBQS, part of which is shown as squares. The figures also show the distorted EW distribution for quasars that are lensed by stars in the disk (dot-dashed line), disk + bulge (dashed line), and disk + bulge + halo (dotted line). Figure 3 corresponds to our model galaxy being at a low redshift $z_{\text {abs }}=0.5$, and with an observed column density $N=5 \times 10^{20} \mathrm{~cm}^{-2}$ (Fig. $3 a$ ) or $N=10^{21} \mathrm{~cm}^{-2}$ (Fig. $3 b$ ). Figure 4 shows the results for $z_{\text {abs }}=2.5$ with $N=5 \times 10^{21}$ $\mathrm{cm}^{-2}$ (Fig. $4 a$ ) or $N=9 \times 10^{21} \mathrm{~cm}^{-2}$ (Fig. $4 b$ ). Note that the area below the curves is in some cases smaller than unity, because we restrict our attention to systems with $\kappa_{*}<1$. In all cases, the distortion of the EW distribution is more pronounced for galaxies with MACHOs. Therefore, the level of this distortion can be used as a sensitive probe of the composition of galactic halos at high redshifts and, by that, complement searches for MACHOs in the local universe (Alcock et al. 1997 and references therein).

In order to estimate the minimum size of the quasar sample that allows detection of the distortion to the EW distribution, we performed a numerical simulation of random deviates drawn from the lensed (i.e., distorted) distribution and computed the $\chi^{2}$ statistic with respect to the observed data for the unlensed distribution (Francis 1993). We assumed that the number of quasars with DLAs is much smaller than the number of quasars without DLAs, which were used to define the unlensed distribution. In this case, $\chi^{2}=\sum_{i}\left[\left(\hat{n}_{i}-n_{i}\right)^{2} / n_{i}\right]$, where $\hat{n}_{i}$ is the number of simulated events from the lensed distribution in the $i$ th bin and $n_{\mathrm{i}}$ is the number of expected events according to the unlensed distribution. For low-redshift $\left(z_{\text {abs }} \sim 0.5\right)$ absorbers with halos made of MACHOs and observed column densities $N \sim 10^{21} \mathrm{~cm}^{-2}$, we find that a relatively small sample of $\sim 10$ systems drawn from the lensed distribution can be rejected as being drawn from the unlensed distribution with a $\chi^{2}$ probability of $\lesssim 5 \times 10^{-2}$.
If MACHOs are absent, the number of systems required to get the same confidence level is increased to $\sim 20$. For high-redshift $\left(z_{\text {abs }} \sim 2.5\right)$ absorbers with MACHOs and $N \sim 10^{21} \mathrm{~cm}^{-2}$, the required number of systems, $\sim 100$, exceeds the number of all known DLAs. However, for highredshift absorbers with column densities on the order of $\sim(8-10) \times 10^{21} \mathrm{~cm}^{-2}$, even a small sample of $\sim 10$ systems could be sufficient to detect the signal at the same confidence level.

We note that our calculations were formulated in terms of the stellar surface density $\kappa_{*}$, and therefore we ignored contributions to the magnification distribution from shear $\gamma$, or additional sources of $\kappa$ (e.g., due to a smoothly distributed dark matter component). The associated increase in the average magnification $\langle\mu\rangle$ would affect both the lines and the continuum. In addition, the finite source size would tend to reduce the expected signal relative to our pointsource calculation. In reality, the magnification is limited to a value $\mu_{\max } \sim r_{\mathrm{E}} / r_{s}$ for $r_{s} \ll r_{\mathrm{E}}$ (see eq. [24]). For a stellar mass of $\sim 0.3 M_{\odot}$ and a continuum-emitting region of a quasar of size $\sim 10^{15} \mathrm{~cm}$, we find $\mu_{\max } \sim 10^{2}$. The contribution to $P(\mu)$ from higher values of $\mu$ is negligible; $P(\mu \gtrsim 100) \lesssim 10^{-7}$ for $\kappa_{*}=0.1$, and $P(\mu \gtrsim 100) \lesssim 10^{-4}$ for $\kappa_{*}=0.8$.

Our calculation of the EW distribution ignores any magnification bias due to lensing, by which faint QSOs are raised above the detection limit. This bias could affect our analysis only if the EW were to depend on the luminosity of the source. Such a dependence, otherwise known as the "Baldwin effect" (Baldwin 1977; Baldwin et al. 1988), is generally very weak and was only marginally detected for the C IV line (Francis 1993). By modeling EW $\propto L^{-0.1}$, this weak scaling results in a $\sim 1 \%$ change of the $\mathrm{EW}$ values (due to magnification bias) for $\kappa_{*}=0.1$ and in a $\sim 5 \%$ change for $\kappa_{*}=0.8$. Thus, the magnification bias can be safely ignored in our calculation.

\subsection{Time Variability}

The microlensing signature discussed in the previous subsection can be detected at one point in time, since it only requires a statistical comparison between the EW distribution of quasars that have DLAs in their spectra and the EW distribution of quasars that do not. By monitoring a quasar over a sufficiently long period of time, it is possible to search for yet another signature of microlensing, namely, an excess temporal variability of the quasar flux due to the motion of the microlenses relative to the quasar. We quantify the probability for detecting this excess variability as a function of the observed $\mathrm{H}$ I column density in our model galaxy.

The net motion of the stars relative to the quasar is the sum of their motion within the galaxy and the bulk velocity of the galaxy relative to the line of sight to the quasar. Wambsganss \& Kundić (1995) have calculated the probability distribution for observing an event with duration smaller than the crossing time of the stellar Einstein radius $t_{0}$ as a function of $\kappa_{*}$ for a population of single-mass stars. Given the source and lens redshifts, the value of $t_{0}$ depends on the mass $M_{\text {star }}$ of the lensing star and on its transverse velocity $v$ relative to the line of sight,

$$
t_{0}=\frac{\sqrt{\left(4 G M_{\mathrm{star}} / c^{2}\right)\left(D_{d} D_{\mathrm{ds}} / D_{s}\right)}}{v},
$$

while an "event" was defined as an increase in the magnification by a factor $\Delta m \gtrsim 0.2 \mathrm{mag}$. Wambsganss \& Kundić 

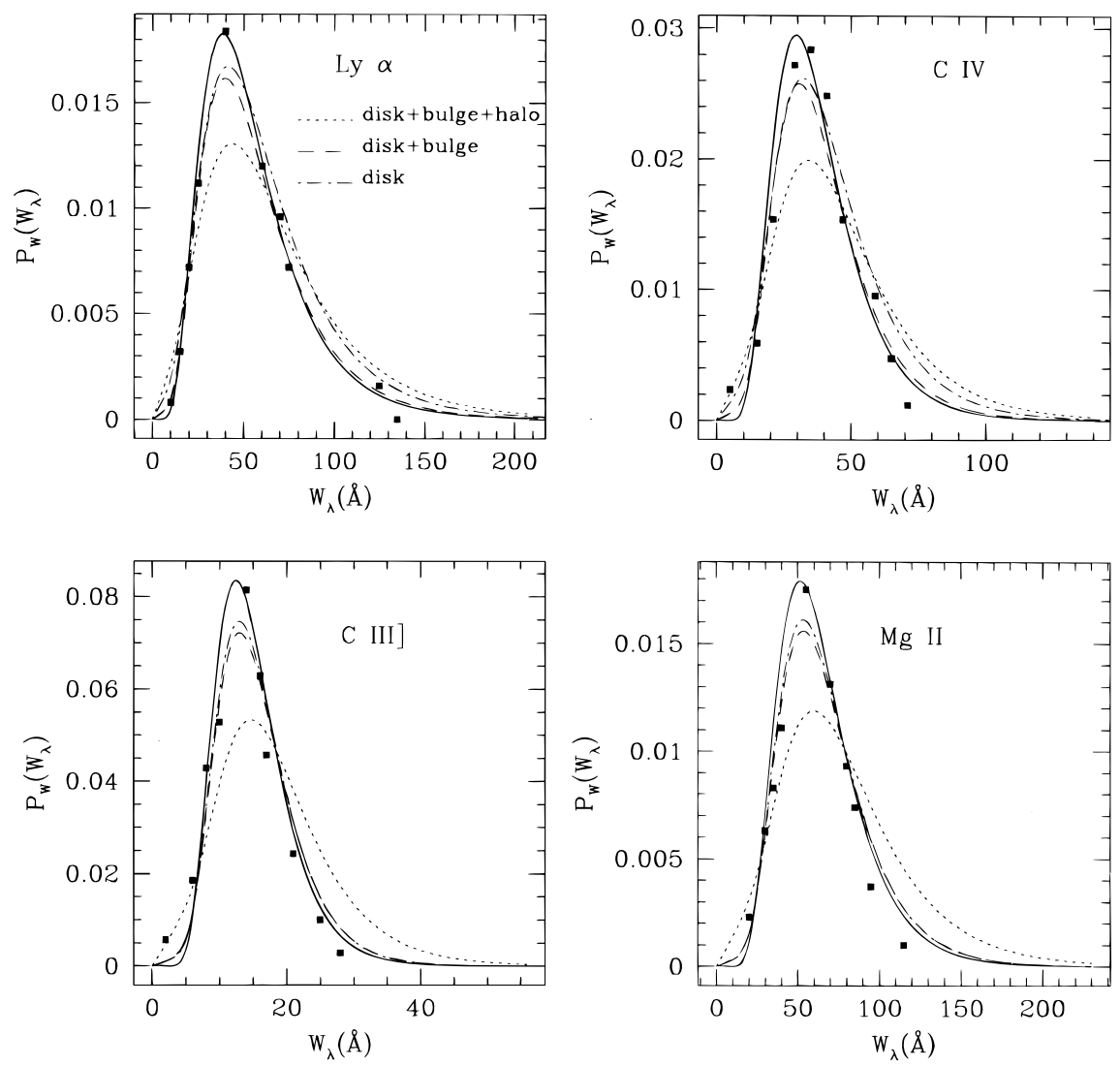

FIG. $3 a$
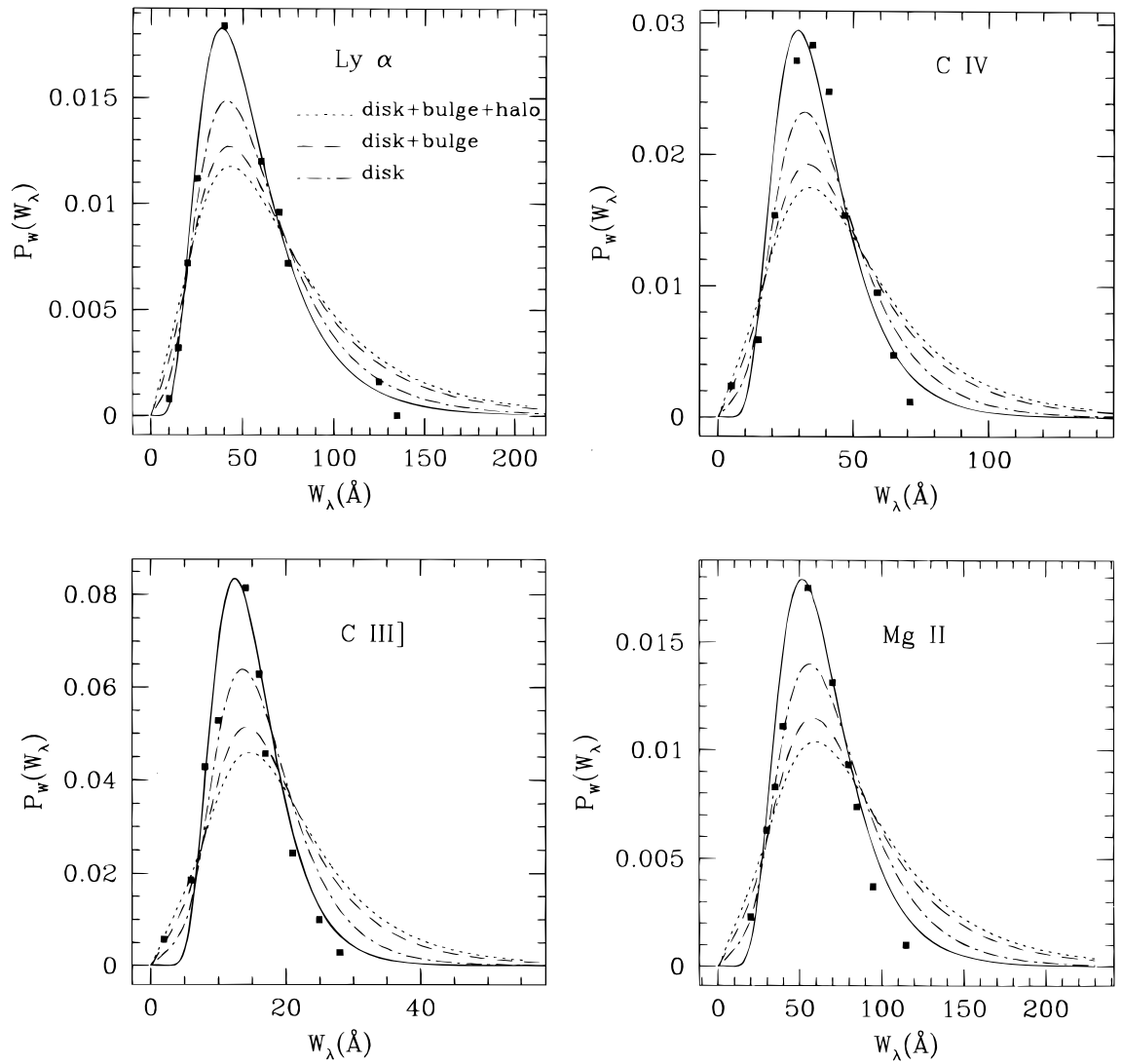

Fig. $3 b$

FIG. 3.- (a) Effect of microlensing on the EW distribution of quasars with DLAs. The solid line shows the analytical log Gaussian fit to the observational data (squares) based on the LBQS (Francis 1993). The other lines show the distortion of this distribution due to microlensing by stars in the disk (dot-dashed line), the disk + bulge (dashed line), and the disk + bulge + halo (dotted line) of our model galaxy. The galaxy is assumed to be observed with an $\mathrm{H}$ i column density $N=5 \times 10^{20} \mathrm{~cm}^{-2}$ and a redshift $z_{\text {abs }}=0.5$. (b) Same as (a), but with $N=10^{21} \mathrm{~cm}^{-2}$. The source redshift is $z_{s}=4$. 

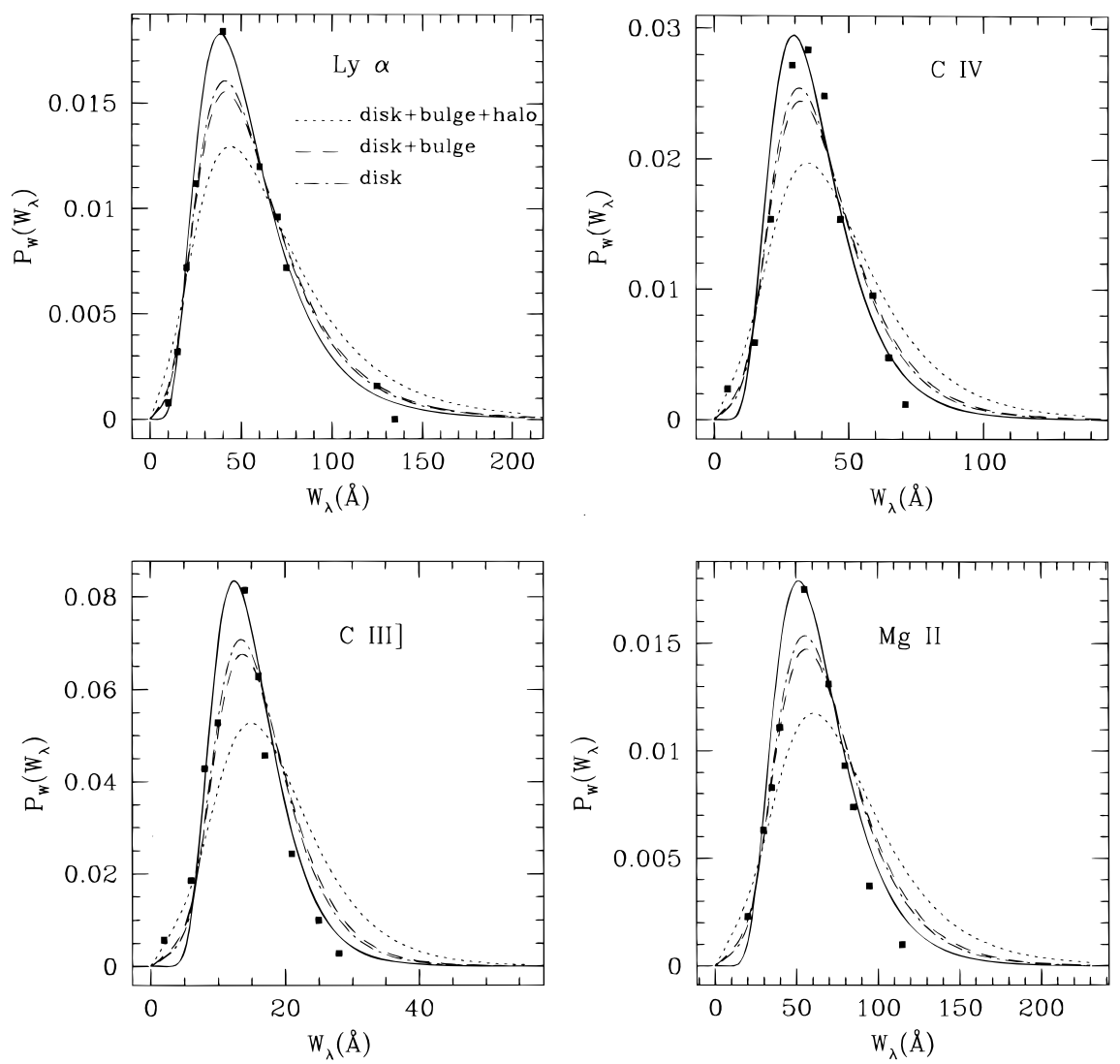

FIG. $4 a$
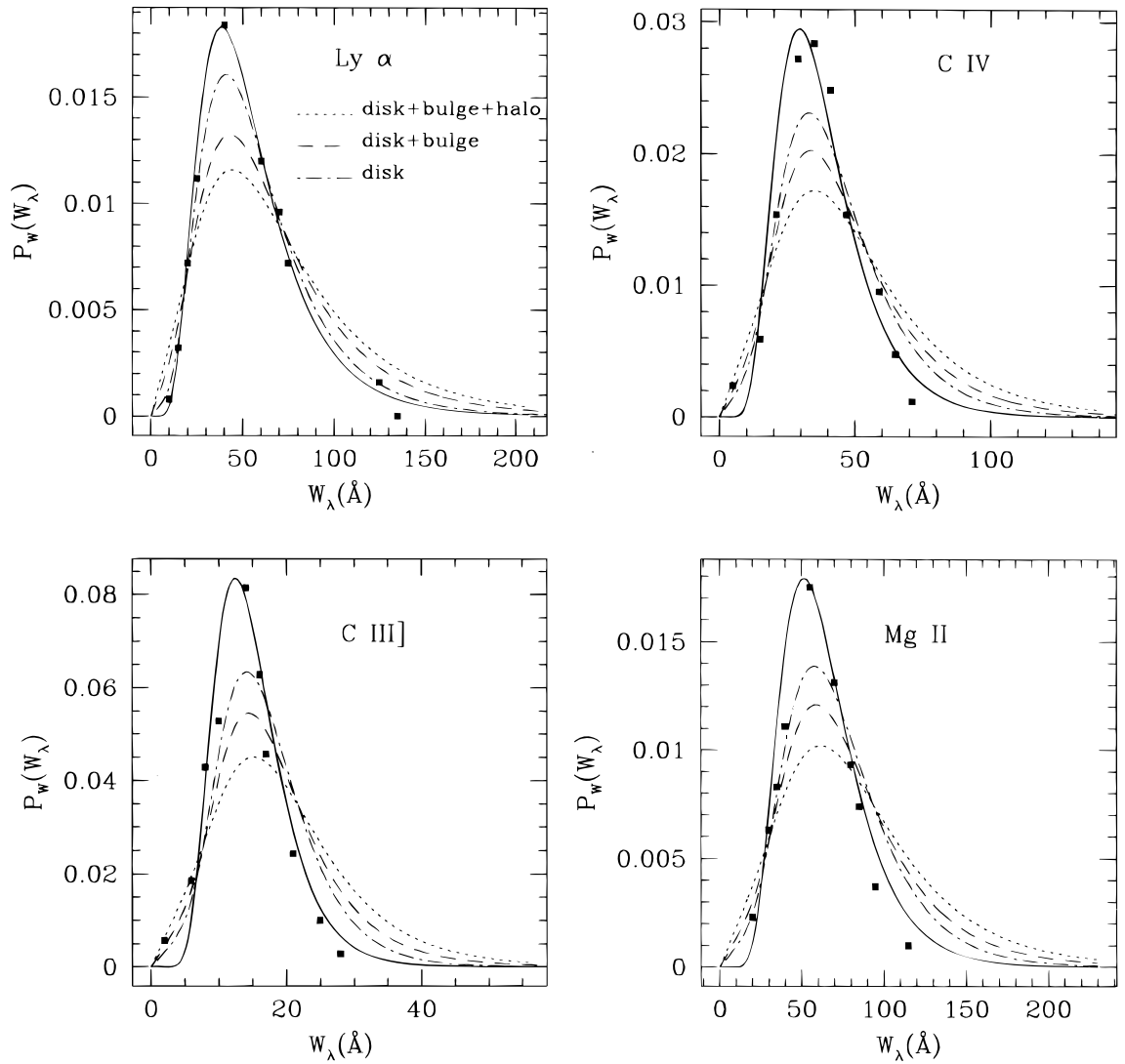

FIG. $4 b$

FIG. 4. (a) Same as Fig. $3 a$, but with $z_{\text {abs }}=2.5$ and $N=5 \times 10^{21} \mathrm{~cm}^{-2}$. (b) Same as (a), but with $N=9 \times 10^{21} \mathrm{~cm}^{-2}$. 
(1995) presented the probability distribution of $t_{0}$ for simulations with $\kappa_{*}=0.2$ in their paper, and they have kindly provided us with their results for $\kappa_{*}=0.5$ and $\kappa_{*}=0.8$ also (J. Wambsganss \& T. Kundić 1996, private communication). In order to obtain a rough estimate for the probability distribution $P\left(t \leq t_{\max } \mid \kappa_{*}\right)$, we have interpolated these numerical results over the entire range of $0 \leq$ $\kappa_{*}<1$. Then, by combining this distribution with equation (14) or equation (23), we have obtained the probability for observing a microlensing event with duration less than $t_{\max }$ given that the observed $\mathrm{H}$ I column density is $N$,

$$
P\left(t \leq t_{\max } \mid N\right)=\int_{0}^{1} d \kappa_{*} P\left(t \leq t_{\max } \mid \kappa_{*}\right) P\left(\kappa_{*} \mid N\right) .
$$

The transverse velocities of stars in galaxies are typically of order $150-200 \mathrm{~km} \mathrm{~s}^{-1}$. The characteristic transverse velocity of galaxies due to large-scale structure can be as large as $500 \mathrm{~km} \mathrm{~s}^{-1}$ (Strauss \& Willick 1995 and references therein). We therefore parameterize, $v=300 v_{300} \mathrm{~km} \mathrm{~s}^{-1}$. The simulation of Wambsganss \& Kundić (1995) assumes a single mass for all stars. We estimate the numerical value of $t_{0}$ by averaging over a Scalo (1986) mass function for the stars, with $\left\langle M^{1 / 2}\right\rangle \approx 0.63 M_{\odot}^{1 / 2}$. Using a source redshift $z_{s}=4$, we then get $t_{0} \approx 28 v_{300}^{-1}$ yr for a lens at $z_{\text {abs }} \approx 0.5$, and $t_{0} \approx 17 v_{300}^{-1} \mathrm{yr}$ at $z_{\mathrm{abs}} \approx 2.5$. Note that the above timescales are averaged over the stellar mass function. In reality, the low-mass tail of the stellar distribution could give rise to events of much shorter duration.

Our results are shown in Figure 5 (for disk + bulge) and Figure 6 (disk + bulge + halo) for a low-redshift absorber (Fig. $6 a$ ) or a high-redshift absorber (Fig. 6b). The figures show the probability of observing a microlensing event with duration less than $t_{\max }=0.08 t_{0}$ (solid line), $0.12 t_{0}$ (dotted line), $0.16 t_{0}$ (dashed line), and $0.2 t_{0}$ (dot-dashed line).

The variable magnification during a microlensing event could be detected through two effects:

1. Changes in the EW of the broad emission lines with time: A magnification factor of $\Delta m \gtrsim 0.2 \mathrm{mag}$ will reduce the EW by a factor $\gtrsim 0.2$ over time. This change could be detected through a monitoring program of quasar emission lines, of the type realized in reverberation studies of quasar variability (Kaspi et al. 1996). It should be generally distinguishable from changes due to intrinsic variability, where an increase in the continuum intensity is followed, after a certain time, by a corresponding increase in the intensity of the emission lines (Maoz 1997).

2. Variability in the QSO light curve: In principle, excess variability in the quasar luminosity due to microlensing could be distinguished from intrinsic quasar variability. In the low optical depth regime $(\kappa \ll 1)$, the microlenses are isolated, and the light curves they produce have generic shapes that are symmetric in time (Paczyński 1986). Even at

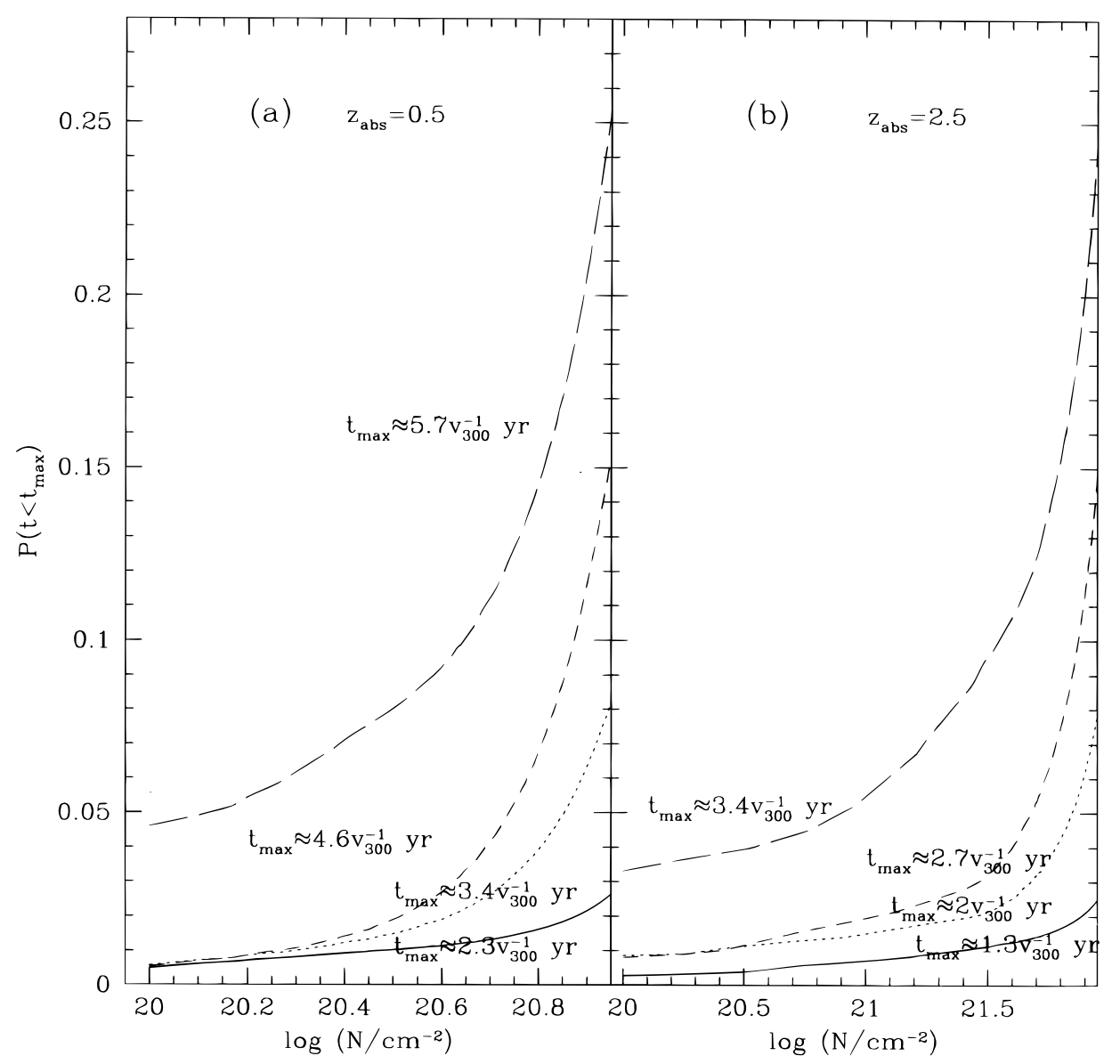

FIG. 5.-Probability for observing a magnification of $0.2 \mathrm{mag}$ with duration shorter than $t_{\max }$ as a function of the observed $\mathrm{H} \mathrm{I} \mathrm{column} \mathrm{density.} \mathrm{Our} \mathrm{model}$ galaxy is located at a redshift $(a) z_{\text {abs }}=0.5$ and $(b) z_{\text {abs }}=2.5$. In both panels, $t_{\max }=0.08 t_{0}$ (solid line), $t_{\max }=0.12 t_{0}$ (dotted line), $t_{\max }=0.16 t_{0}$ (short-dashed line), and $t_{\max }=0.2 t_{0}$ (long-dashed line). Here $t_{0} \approx 28 v_{300}^{-1} \mathrm{yr}$ at $z_{\mathrm{abs}}=0.5$, and $t_{0} \approx 17 v_{300}^{-1} \mathrm{yr}$ at $z_{\mathrm{abs}}=2.5$, where $v_{300}$ is the transverse velocity of the microlenses relative to the source in units of $300 \mathrm{~km} \mathrm{~s}^{-1}$. These numbers were obtained for the average stellar mass in a Scalo (1986) mass function. The source redshift is fixed at $z_{s}=4$. 


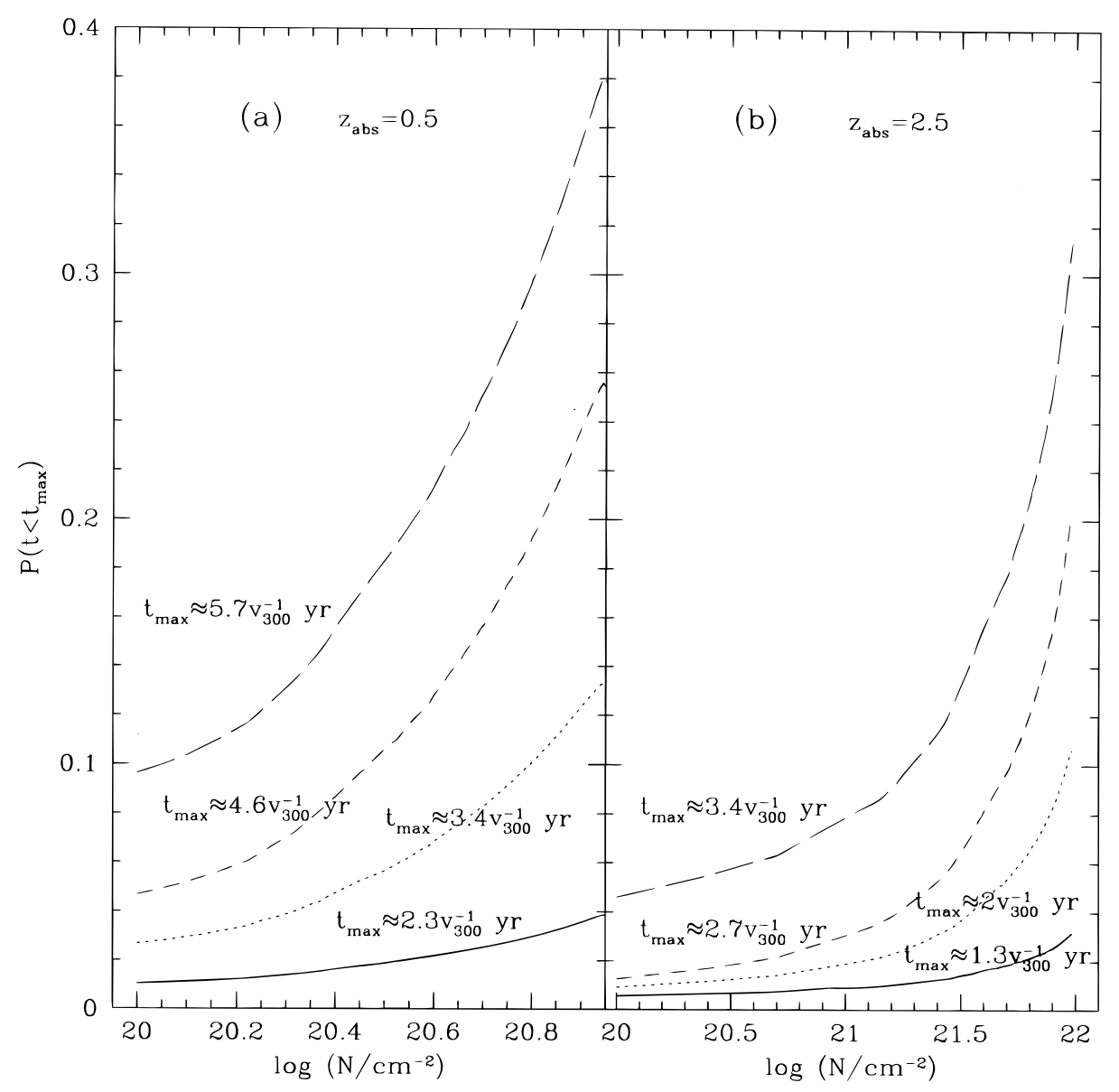

FIG. 6.-Same as Fig. 5, but including a halo made of MACHOs

higher optical depths, there should still be a statistical symmetry between the rising and the falling parts of the light curves. Finally, as long as the continuum emission region is much smaller than the size of the stellar lensing zone, the excess temporal variation of the quasar should be achromatic.

\section{CONCLUSIONS}

We have shown that the subset of all quasars that have damped $\mathrm{Ly} \alpha$ absorption in their spectra due to an intervening spiral galaxy should possess an enhanced tail in the EW distribution of their broad emission lines, and exhibit excess variability, relative to the rest of the quasar population. Both of these microlensing signatures are more pronounced if the halos of DLAs are composed of compact objects.

Previous attempts to find a redshift evolution in the EW distribution of a large sample of quasars due to microlensing by a population of intergalactic stars have yielded a negative result (Dalcanton et al. 1994). In this paper, we have shown that the significance of microlensing relative to the statistical noise should be much more pronounced in a subset of all quasars that are located behind galactic $\mathrm{H} \mathrm{I}$ disks. Using a simple closed box model for a spiral galaxy, we have found that disk + bulge microlensing could be detected through its imprint on the equivalent width distortion with a signal-to-noise ratio $\gtrsim 2$ in a sample of $\approx 20$ DLAs at $z_{\text {abs }} \lesssim 1$ with $N \sim 10^{21} \mathrm{~cm}^{-2}$. The necessary sample size is reduced to $\approx 10$ if the galaxy halo is com- posed of MACHOs with a velocity dispersion of $\sigma \approx 170$ $\mathrm{km} \mathrm{s}^{-1}$. The necessary sample size should scale as proportional to $\sigma^{-4}$ for other galactic systems, and therefore massive galaxies are likely to dominate the statistics. In addition, we find that about one-tenth of all quasars with DLAs are likely to show excess variability on timescales shorter than 5 yr (cf. Figs. 5 and 6).

Unfortunately, the current sample of $\sim 80$ quasars with DLAs (Wolfe et al. 1995) includes only several absorbers with $z \lesssim 1$ and might not be sufficiently large to demonstrate the existence of microlensing. The number of known DLAs could increase by an order of magnitude as a result of spectroscopic follow-ups on the catalog of $\sim 10^{5}$ quasars that is expected to be compiled by the forthcoming Sloan Digital Sky Survey (Gunn \& Knapp 1993). Even before any absorption data is reduced, it would be interesting to select background quasars that are projected close to foreground galaxies and therefore are likely to be microlensed. Related studies by Webster et al. (1988) show an increased number of quasar-galaxy pairs than expected from random alignments. The enhancement in the quasar surface density near galaxies was interpreted as macrolensing and was used to draw conclusions about the distribution of matter around galaxies.

Detection of the level of microlensing for quasars with damped Ly $\alpha$ absorption can be used to calibrate the mass fraction in the form of massive compact objects in galactic halos at high redshifts. Figures 3-6 show that the microlensing signal is enhanced when an isothermal halo made of 
MACHOs is added to the galactic disk and bulge. Direct imaging of the DLAs can be used to infer the projected separation between the luminous center of the absorber and the quasar. In cases where the DLA redshift is known (e.g., through its Ly $\alpha$ emission; see Djorgovski et al. 1996 and Lu et al. 1997), it also might be possible to infer spectroscopically the velocity dispersion of the intervening galaxy. When combined with the information gathered by microlensing searches in the local universe, such studies could extend our knowledge of the composition of galactic halos out to redshifts as high as $z \sim 5$.

We thank an anonymous referee for insightful comments that improved the presentation. This work was supported in part by the NASA ATP grant NAG 5-3085 and the Harvard Milton fund (for A. L.), and by a fellowship from the University of Salerno, Italy (for R. P.).
Alcock, C, et al. 1997, ApJ, 479, 119

Baldwin, J. A. 1977, ApJ, 214, 679

Baldwin, J. A., McMahon, R., Hazard, C., \& Williams, R. E. 1988, ApJ, 327,103

Bartelmann, M \& Loeb, A. 1996, ApJ, 457, 529

Briggs, F. H., Brinks, E., \& Wolfe, A. M. 1997, AJ, 113, 467

Briggs, F. H., Wolfe, A. M., Liszt, H. S., Davis, M. M., \& Turner, K. L. 1989, ApJ, 341, 650

Broeils, A. H., \& Courteau, S. 1997, in ASP Conf. Ser. 117, Dark and Visible Matter in Galaxies and Cosmological Implications, ed. M. Persic \& P. Salucci (San Francisco: ASP), in press (astro-ph/9610264)

Broeils, A. H., \& van Woerden, H. 1994, A\&AS, 107, 129

Burstein, D. 1979, ApJS, 41, 435

Canizares, C. R. 1982, ApJ, 263, 508

Dalcanton, J. J., Canizares, C. R., Granados, A., \& Stocke, J. T. 1994, ApJ, 568,424

Djorgovski, S. G., Pahre, M. A., Bechtold, J., \& Elston, R. 1996, Nature, 382,234

Eisenstein, D. J., \& Loeb, A. 1996, ApJ, 459, 432

Fall, S. M., \& Pei, Y. C. 1996, ApJ, 464, L43

Francis, P. J. 1993, ApJ, 405, 119

Freeman, K. C. 1970, ApJ, 160, 811

Gould, A., \& Miralda-Escudé, J. 1996, preprint (astro-ph/9612144)

Gunn, J. E., \& Knapp, J. 1993, in ASP Conf. Ser. 43, Sky Surveys: Protostars to Protogalaxies, ed. B. T. Soifer (San Francisco: ASP), 267

Hawkins, M. R. S. 1996, MNRAS, 278, 787

Irwin, M. J., Webster, R. L., Hewett, P. C., Corrigan, R. T., \& Jedrzejewski, R. I. 1989, AJ, 98, 1989

Kaspi, S., Smith, P. S., Maoz, D., Netzer, H., \& Jannuzi, B. T. 1996, ApJ, 471, L75

Kundić, T., \& Wambsganss, J. 1993, ApJ, 404, 455

Lanzetta, K. M., Wolfe, A. M., \& Turnshek, D. A. 1995, ApJ, 440, 435

Laor, A. 1990, MNRAS, 246, 369

Le Brun, V., Bergeron, J., \& Boissé, P. 1996, A\&A, 306, 691

Lewis, G. F., Miralda-Escude, J., Richardson, D. C., \& Wambsganss, J. 1993, MNRAS, 261, 647

Loeb, A. 1997, in Texas Symp., in press (astro-ph/9701100)

Lowenthal, J. D., et al. 1997, ApJ, 481, 673

Lu, L., Sargent, W. L. W., \& Barlow, T. A. 1997, ApJ, 484, 131

Lu, L., Sargent, W. L. W., Barlow, T. A., Churchill, C. W., \& Vogt, S. S. 1996, ApJS, 107, 475

Lu, L., \& Wolfe, A. M. 1994, AJ, 108, 44

Lu, L., Wolfe, A. M., Turnshek, D. A., \& Lanzetta, K. M. 1993, ApJS, 77,

Madau, P. 1996, preprint (astro-ph/9612157)

Mao, S., \& Kochanek, C. 1994, MNRAS, 268, 569

Maoz, D. 1997, in ASP Conf. Ser. 113, Emission Lines in Active Galaxies: New Methods and Techniques, IAU Colloq. 159, ed. B. M. Peterson,

F.-Z. Cheng, \& A. S. Wilson (San Francisco: ASP), in press (astro$\mathrm{ph} / 9609174)$

Nemiroff, R. J. 1988, ApJ, 335, 593

Paczyński, B. 1986, ApJ, 304, 1

Peacock, J. A. 1986, MNRAS, 223, 113

\section{EFERENCES}

Pei, Y. C., Fall, S. M., \& Bechtold, J. 1991, ApJ, 378, 6

Perna, R., Loeb, A., \& Bartelmann, M. 1997, ApJ, 488, 550

Perry, J. J., Watson, A. M., \& Kronberg, P. P. 1993, ApJ, 406, 407

Peterson, B. M. 1993, PASP, 105, 247

Pettini, M., King, D. L., Smith, L. J., \& Hunstead, R. W. 1997, ApJ, 478, 536

Pettini, M., Smith, L. J., Hunstead, R. W., \& King, D. L. 1994, ApJ, 426, 79

Prochaska, J. X., \& Wolfe, A. M. 1997, ApJ, 474, 140

Racine, R. 1991, AJ, 102, 454

Rauch, K. P., \& Blandford, R. D. 1991, ApJ, 381, L39

Rauch, K. P., Mao, S., Wambsganss, J., \& Paczyński, B. 1992, ApJ, 386, 30

Refregier, A., \& Loeb, A. 1997, ApJ, 478, 476

Rix, H.-W., Maoz, D., Turner, E., \& Fukugita, M. 1994, ApJ, 435, 49

Scalo, J. M. 1986, Fund. Cosmic Phys., 11, 1

Schneider, P., Ehlers, J., \& Falco, E. E. 1992, Gravitational Lenses (Heidelberg: Springer)

Smith, L. J., Pettini, M., King, D. L., \& Hunstead, R. W. 1996, preprint (astro-ph/9601153)

Steidel, C. C., Bowen, D. V., Blades, J. C., \& Dickenson, M. 1995, ApJ, 440, L455

Steidel, C. C., Giavalisco, M., Pettini, M., Dickinson, M., \& Adelberger, K. L. 1996, AJ, 112, 352

Steidel, C. C., Pettini, M., Dickinson, M., \& Persson, S. E. 1994, AJ, 108, 2046

Storrie-Lombardi, L. J., Irwin, M. J., \& McMahon, R. G. 1996a, MNRAS, 282,1330

Storrie-Lombardi, L. J., McMahan, R. G., Irwin, M. J., \& Hazard, C. 1996b, ApJ, 468, 121

Strauss, M. A., \& Willick, J. A. 1995, Phys. Rep., 261, 271

Tóth, G., \& Ostriker, J. P. 1992, ApJ, 389, 5

Turnshek, D. A., Wolfe, A. M., Lanzetta, K. M., Briggs, F. H., Cohen, R. D., Foltz, C. B., Smith, H. E., \& Wilkes, B. J. 1989, ApJ, 344, 567

Vogt, N. P., Forbes, D. A., Phillips, A. C., Gronwall, C., Faber, S. M., Illingworth, G. D., \& Koo, D. C. 1996, ApJ, 465, L15

Wambsganss, J. 1990, Ph.D. thesis, Ludwig-Maximilians-Universitaet Munich

Wambsganss, J., \& Kundić, T. 1995, ApJ, 450, 19

Wambsganss, J., Paczyński, B., \& Schneider, P. 1990, ApJ, 358, L33

Webster, R. L., Hewett, P. C., Harding, M. E., \& Wegner, G. A. 1988, Nature, 336, 358

Welter, G. L., Perry, J. J., \& Kronberg, P. P. 1984, ApJ, 279, 19

Witt, H. J. 1993, ApJ, 403, 530

Wolfe, A. M. 1995, in QSO Absorption Lines, ed. G. Meylan (Heidelberg: Springer), 13

Wolfe, A. M., Lanzetta, K. M., Foltz, C. B., \& Chaffee, F. H. 1995, ApJ, 454, 698

Wolfe, A. M., Lanzetta, K. M., \& Oren, A. L. 1992a, ApJ, 388, 17

Wolfe, A. M., Turnshek, D. A., Lanzetta, K. M., \& Lu, L. 1993, ApJ, 404, 480

Wolfe, A. M., Turnshek, D. A., Lanzetta, K. M., Lu, L., \& Oke, J. B. 1992b, ApJ, 385, 151

Zaritzky, D., \& Rix, H.-W. 1997, ApJ, 477, 118 